\title{
PERTURBATIONS NEAR ZERO \\ OF THE LEADING COEFFICIENT OF SOLUTIONS TO A NONLINEAR DIFFERENTIAL EQUATION
}

\author{
VADIM KOMKOV
}

ABSTRACT. The equation

$$
\frac{d}{d t}\left(a(t) \cdot \psi(x) \frac{d x}{d t}\right)+f(x) \cdot \frac{d x}{d t}+c(t) x=g(t)
$$

that generalizes the more commonly studied selfadjoint second order equation

$$
\frac{d}{d t}\left(a(t) \frac{d x}{d t}\right)+c(t) x=g(t)
$$

occurs in celestial dynamics, in the study of gyroscopic systems, and in other problems of nonlinear mechanics. Using techniques of nonstandard analysis we derive some properties of the "duck"-type cycles for the solutions of this equation.

1. Some basic symbolism and notation of nonstandard analysis. $R$ will denote the reals, and ${ }^{*} R$ a nonstandard extension of $R$ which is at least $\boldsymbol{\kappa}_{0}$ saturated. For any measurable subset $\Omega$ of $R$ (with respect to the Borel measure) * $\Omega$ will denote the corresponding subset of ${ }^{*} R$, i.e. the scope of $\Omega$ in ${ }^{*} R$. If $\Omega$ is a countable set, we can introduce the Fréchet filter $F$ of all of all subsets of $\Omega$ whose complement is finite. Let $U$ be an ultrafilter containing $F$. If $U$ is a free ultrafilter, the Loś construction may be used to produce a nonstandard extension of $R$ by generating appropriate equivalence classes with the natural embedding of $R$ in ${ }^{*} R$. The symbol ${ }^{*} R_{\mathrm{Bd}}$ will denote the near-standard part of ${ }^{*} R$, i.e. the subset of ${ }^{*} R$ such that $x \in{ }^{*} R_{\mathrm{Bd}}$ implies that $x$ lies in the monad of some standard number ${ }^{0} x \in R .{ }^{*} R_{\infty}$ denotes the complement of ${ }^{*} R_{\mathrm{Bd}}$ in ${ }^{*} R$. The natural numbers are denoted by $N$, and ${ }^{*} N$ denotes a nonstandard extension of $N$.

The equivalence relation (being close to) is introduced on ${ }^{*} R . x \approx y$ implies that $|x-y|$ is an infinitesimal, i.e. either $x-y=0$, or else $x-y$ is an inverse of some number in ${ }^{*} R_{\infty}$. Since $R$ and ${ }^{*} R$ are models for the same set of sentences, the transfer theorem can be applied. Any well-formed sentence in the language $L$ (formed recursively) that is true in $R$ is also true in ${ }^{*} R$, and if it is false in $R$ then it

Received by the editors June 19, 1985 and, in revised form, November 1, 1985. The contents of this paper were presented to the American Mathematical Society at the meeting held at the University of Illinois in Chicago on March 22, 1985.

1980 Mathematics Subject Classification (1985 Revision). Primary 34C05, 34C28, 03H10; Secondary $58 \mathrm{~F} 22$.

This research is supported by NSF Grant DMC 84-16155. 
is false in ${ }^{*} R$. Conversely, a sentence concerning internal sets (or internal functions and relations) that is true in ${ }^{*} R$ is also true in $R$, and if it is false in ${ }^{*} R$ then it is false in $R$. For an introduction to nonstandard analysis see notes of Luxemburg [6] or "the bible", i.e. the monograph of Abraham Robinson [5].

2. Poincarés equations of motion. In the study of nonlinear mechanics, one encounters the basic one-dimensional Hamiltonian system discussed in this article, that either describes the motion in a central force field or in a two body problem where one body may have a variable mass.

Let us consider a planar motion of a point mass in the usual $(r, \theta)$ polar coordinates.

The angular momentum for the variable mass $m(t)$ is given by

$$
p=m(t) r^{2}(t) \dot{\theta}(t) .
$$

The "canonical equation" (see for example [1]) $\partial H / \partial \theta=\dot{p}_{\theta}$ assumes the form

$$
\frac{d}{d t}\left[m(t) r^{2} \frac{d \theta}{d t}\right]=\frac{\partial H}{\partial \theta}=-c(t) \cdot y(r, \theta)
$$

Eliminating $r$ by solving the "radial canonical equation" $\partial H / \partial r=\dot{p}_{r}$, and substituting, where necessary, the other "canonical" relations

$$
\partial H / \partial p_{\theta}=-\dot{\theta}, \quad \partial H / \partial p_{r}=-\dot{r},
$$

we may derive a relation $r=\psi(\theta)$.

Generally, in such problems, it is not possible to solve explicitly for $\theta$ as function of $r$. For example, in the physical interpretation of a motion in a central force field the angular variable $\theta$ is frequently a multivalued function of $r$.

Substituting the relation $r=\psi(\theta)$ into equation (2) we obtain

$$
\frac{d}{d t}\left(m(t)(\psi(\theta))^{2} \frac{d \theta}{d t}\right)+c(t) f(\theta)=0 .
$$

where $f(\theta)=y(\psi(\theta), \theta)$. This equation has been studied by Poincaré [1] and by the author in [2 and 3]. Under some conditions the system (3) displays the fast-slow behavior that is the main topic of this work.

3. Some examples. One such example concerns the motion of a gyroscope.

The motion of a gyroscope or a spinning top can be written as Euler's equation

$$
\frac{d}{d t}\left(\frac{\partial T}{\partial \underset{\sim}{\dot{q}}}\right)-\frac{\partial T}{\partial q}=\underset{\sim}{Q}(\underset{\sim}{q}, t)-\mathbf{B}(\underset{\sim}{q}, t) \underset{\sim}{\dot{q}}-\mathbf{G}(\underset{\sim}{q}, t) \underset{\sim}{\dot{q}}, \quad \text { where } \underset{\sim}{Q}=\frac{\partial V}{\partial q}
$$

$Q$ is the force derived from a potential.

The kinetic energy is a quadratic form

$$
\begin{aligned}
& T=\frac{1}{2} \dot{\sim}^{\tau} \mathbf{A}(\underset{\sim}{q}, t) \underset{\sim}{\dot{q}}, \\
& q=\left(q_{1}, q_{2}, \ldots, q_{n}\right),
\end{aligned}
$$

$\mathbf{A}$ is a positive definite $n \times n$ matrix,

$\mathbf{B}$ is a symmetric matrix of dissipative forces,

$\mathbf{G}$ is a skew-symmetric matrix of gyroscopic forces,

${ }^{\tau}$ denotes the transpose. 
A Legendre transformation introduces a generalized momentum

$$
\underset{p}{p}=\partial L / \partial \dot{q}=A \dot{q} \text {. }
$$

Then

(5) $\underset{\sim}{\dot{p}}=\frac{1}{2}{\underset{\sim}{\tau}}^{\tau}\left(A^{-1}\right)^{\tau} \frac{\partial A}{\partial \underset{\sim}{q}}\left(A^{-1} \underset{\sim}{p}\right)-(B+G) A^{-1} \underset{\sim}{p}+\underset{\sim}{Q}=\frac{\partial W}{\partial \underset{\sim}{q}}-(B+G) A^{-1} \underset{\sim}{p}$,

and

$$
\underset{\sim}{\dot{q}}=A^{-1} \underset{\sim}{p}=\partial W / \partial \underset{\sim}{p}
$$

This becomes a Hamiltonian system if $B=G$, and particularly if $B=G=\varnothing$. In the study of spinning tops and gyroscopes we certainly do not assume that $G=\varnothing$. In fact, the common assumption is that components of $G$ are large compared to components of $B$. Denoting by $\varepsilon$ the quantity $\varepsilon=1 /\|G\|$ it is possible to show that equations (5) and (6) can be approximated by

$$
\begin{aligned}
& \underset{\sim}{\dot{q}}=A^{-1} \underset{\sim}{p}, \\
& \underset{\sim}{\dot{p}}=(\varepsilon B+G) A^{-1} \underset{\sim}{p}+\varepsilon \underset{\sim}{M}+\underset{\sim}{Q}, \\
& M=\frac{1}{2} \underline{\sim}^{\tau}\left(A^{-1}\right)^{\tau}(\partial A / \partial \underset{\sim}{q}) A^{-1} \underline{\sim}
\end{aligned}
$$

(see D. P. Merkin [8]). This is an example of a slow-fast system, discussed in this work. After division by $\varepsilon$ the "canonical" system $\left(7^{\mathrm{a}}\right),\left(7^{\mathrm{b}}\right),\left(7^{\mathrm{c}}\right)$ assumes the following form:

$$
\begin{aligned}
& \underset{\sim}{\dot{q}}=A^{-1} \underset{\sim}{p}, \\
& \underset{\sim}{\dot{p}}=\left(B+\varepsilon^{-1} G\right) A^{-1} \underset{\sim}{p}+\underset{\sim}{M}+\varepsilon^{-1} \underset{\sim}{Q},
\end{aligned}
$$

with $q \in{ }^{*} R^{3}, p \in{ }^{*} R^{3}, \varepsilon \approx 0$. Other examples of slow-fast systems may be found in the papers of Diener [9] and in the recent works of J. Cronin-Scanlon concerning the periodicity of periodically perturbed biological systems of the Hodgkin and Huxley type (see [11] for the original work of Hodgkin and Huxley). Equations of the type

$$
\dot{x}=\frac{1}{\varepsilon} F(x, y)+H(x, y, t), \quad \dot{y}=G(x, y)+\varepsilon K(x, y, t)
$$

were investigated by Cronin-Scanlon (recent developments were announced in the abstract [10]). The question: "Does there exist a periodic solution near a singularity?" was answered "yes" by Levinson in 1957, thus initiating research into cyclic behavior near a singular point. Sibuya and Cronin-Scanlon studied problems of this type. However, a detailed analysis of behavior and sensitivity of such solutions seem to be emerging only after a better understanding of the complexity of the behavior of such systems as $\varepsilon$ becomes literally infinitely small. The most widely investigated slow-fast system is the van der Pol equation

$$
\varepsilon \dot{x}+\left(x^{2}-1\right) \dot{x}+x=0,
$$


describing the current flow through a triode tube. A simple change of variable converts the van der Pol equation into the slow-fast Lienard system of two equations

$$
\dot{x}=f(x, y), \quad \dot{y}=\varepsilon^{-1} g(x, y),
$$

where $f(x, y)$ and $g(x, y)$ are well behaved, that is, $f$ and $g$ are sufficiently smooth functions, while $\varepsilon$ is "very small".

\section{Some "duck" theorems.}

4a. Assumptions. We consider equation (3) with an inhomogeneous term

$$
\frac{d}{d t}\left(a(t) \cdot \psi(x) \frac{d x}{d t}\right)+f(x) \frac{d x}{d t}+c(t) \cdot x=g(t)
$$

making the following assumptions:

$$
\left.\begin{array}{l}
\psi(x)>0 \\
f(x)>0
\end{array}\right\} \text { if } x \neq 0
$$

and

$\left(10^{\mathrm{a}}\right)$

$$
\left\{\begin{array}{l}
\psi, f \in C(-\infty,+\infty) \\
\psi(x) \text { is bounded on }(-\infty, \infty) \\
a(t), c(t) \text { are nonnegative functions on } \Omega \\
a(T) \in C^{1}(\Omega), c(t) \in C(0, \infty), \lim _{t \rightarrow \infty} a(t)=0, \limsup _{t \rightarrow \infty} c(t) \text { exists, } \\
g(t) \in C(\Omega), \\
\lim _{t \rightarrow \infty} g(t) \text { exists }(\Omega=[0, T], \text { where possibly } T=+\infty)
\end{array}\right.
$$

Let

$$
F(x)=\int^{x} f(\xi) d \xi
$$

We denote

$$
y=F(x)+a(t) \cdot \psi(x) \dot{x} \quad(\text { where } \cdot=d / d t) .
$$

Then equation (10) can be rewritten as a system of two equations:

$$
\begin{aligned}
& d y / d t=-c(t) \cdot x+g(t), \\
& d x / d t=a^{-1}(t)[y-F(x) / \psi(x)] .
\end{aligned}
$$

The $\{x, y\}$ variables are the Lienard phase space variables. Analysis in the Lienard plane for the van der Pol equation can be found in several recent articles [7,9]. We consider solutions of the system $\left(13^{\mathrm{a}}\right),\left(13^{\mathrm{b}}\right)$ in the ${ }^{*} R^{2}$ plane. For large values of $t$ we have a system with one slow variable and one fast variable. Clearly if $\dot{x} \notin{ }^{*} R_{\infty}$ then $y=F(x)$.

The local extremal points of $F(x)$, such as $\tilde{x}$, are the candidates for the Hopf bifurcation. This behavior can be clarified by extending the time axis from $R$ to a nonstandard model ${ }^{*} R$, and extending the corresponding values of $x, y$ and their derivatives to ${ }^{*} R$. From now on we shall study the behavior of equations $\left(13^{\mathrm{a}}\right)$ and $\left(13^{\mathrm{b}}\right)$ on the infinite part of the $t$ axis $\left(t \in{ }^{*} R_{\infty}\right)$. 


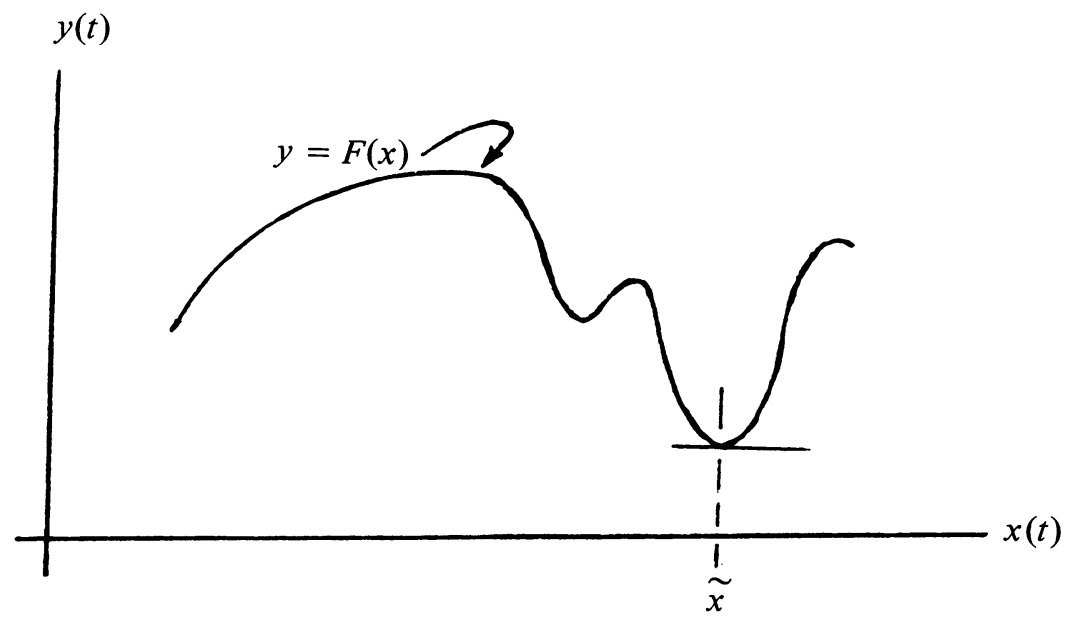

FIGURE 1

Since $c(t)$ and $g(t)$ are bounded by a standard number at any infinite time, $y(t)$ is a slow variable.

$\lim _{t \rightarrow \infty} a(t)=0$ implies that $a^{-1}(t) \in{ }^{*} R_{\infty}$ when $t \in{ }^{*} R_{\infty}$. Hence, for any $x \in{ }^{*} R_{\mathrm{Bd}}, a^{-1}(t) / \psi(x) \in{ }^{*} R_{\infty}$. Therefore, $x$ is a fast variable, except in some infinitesimal neighborhood of the monad of zero of the function $\{y-F(x)\}$, i.e. in the "halo" of $\{y=F(x)\}$.

4b. A preliminary observation. Let us consider the case when either [lim inf $c(t)]>$ 0 , and the motion is contained in the part of the trajectory that does not contain the set $\{x=0\}$, or else [liminf $g(t)>0$ ]. Such assumptions imply that $d y / d t \neq 0$. If any of these assumptions are true then we assert that noninfinitesimal changes in the value of $y(t)$ occur only on finite time intervals.

Proof. Let us refer to the monad of the Lienard curve $\Gamma$ as the "highway". A bounded displacement off the highway corresponds to an infinitesimal time change. Let us consider the behavior of a trajectory on the highway, but not in the monad of a critical point. Since $d x / d t, d y / d t$ are noninfinitesimal near-standard quantities on such part of the trajectory, finite change in $x(t)$ and $y(t)$ on $\left(t_{0}, t_{1}\right)$ corresponds to the statement $\left(t_{1}-t_{0}\right) \in{ }^{*} R_{\mathrm{Bd}}$ and $x\left(t_{1}\right)-x\left(t_{2}\right) \neq 0$ whenever $\left(t_{1}-t_{0}\right) \neq 0$.

We see that infinite changes in time parameter may occur only if "excursions from the highway" take place, or if the trajectory is unbounded.

4c. Some lemmas for bounded trajectories. Let $r(t)$ denote $\|x\|$, f.e. the distance of $x(t)$ from the origin, at the time $t$.

LEMMA 1. For bounded trajectories we can omit the condition $\psi(x) \in{ }^{*} R_{\mathrm{Bd}}$ for all $x \in(-\infty,+\infty)$. It suffices that $\psi(x)$ is a continuous function of $x$ to conclude that $y \approx F(x(t))$ is a necessary condition for $(\dot{x})^{-1} \approx 0$ along the trajectory of motion for equation (10) on any infinite time interval $\left(t_{1}, t_{2}\right), t_{1} \in{ }^{*} R_{\infty}$. 


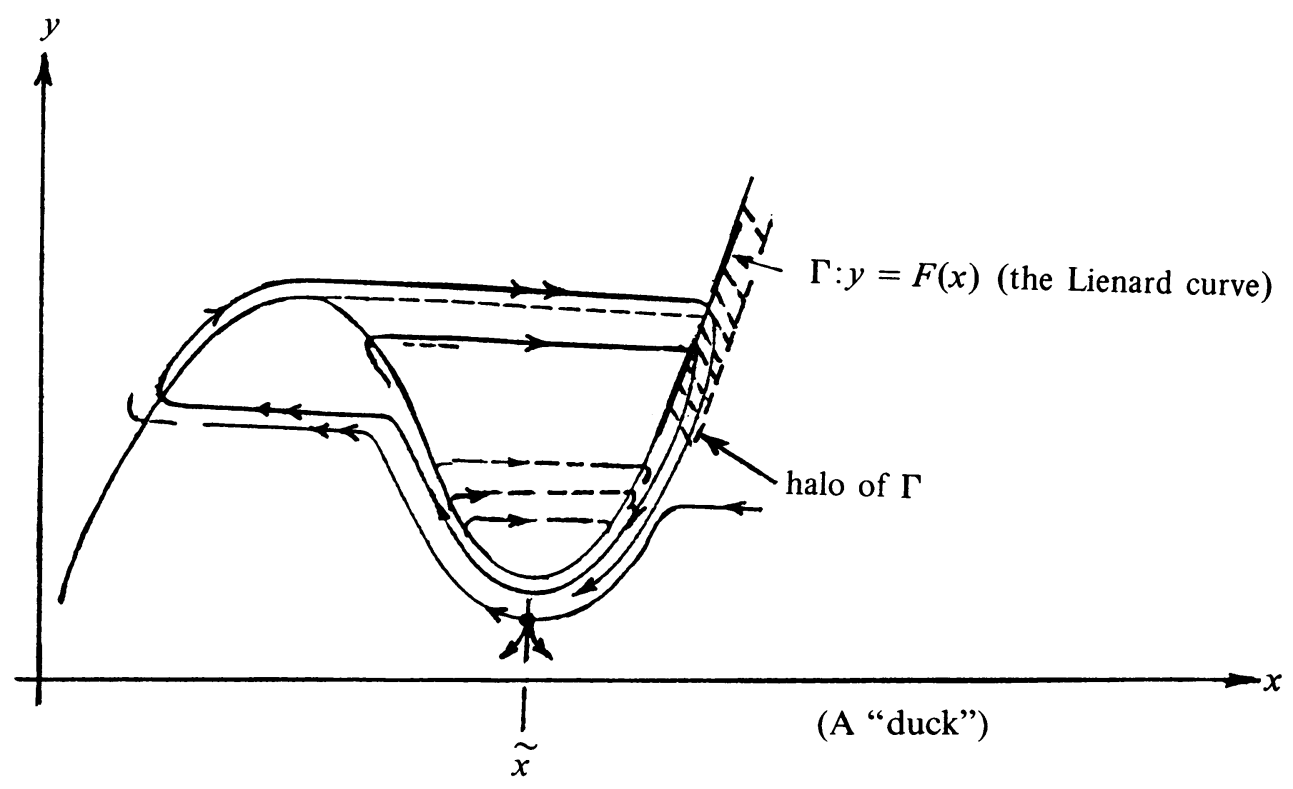

FIGURE 2

Proof. A trajectory is bounded on some time interval if and only if $r(t)$ is near standard on that interval. Hence, $\psi(x(t)) \in{ }^{*} R_{\mathrm{Bd}}$. However, for any $t \in{ }^{*} R_{\infty}$ the function $a^{-1}(t)$ is infinite and so is $a^{-1}(t) / \psi(x)$. Hence (according to equation $\left.(13)^{\mathrm{b}}\right), y(t)-F(x(t))$ must be infinitesimal if $\dot{x}(t)$ is not infinite.

Lemma 2. ("Obedience to Highway Directions" Lemma). If for some $\hat{t} \in{ }^{*} R_{\infty}$ : $\bar{y}(\hat{t}) \approx(F \bar{x}(\hat{t}))$ at some point $(\bar{x}, \bar{y})$ on trajectory of equation (10) that lies on the attractive part of the Lienard curve $\Gamma: y=F(x)$ and $\dot{\bar{y}} / \dot{\bar{x}} \approx d y /\left.d x\right|_{\Gamma}$ then $y(\bar{t}) \approx$ $F(\bar{x}(\bar{t}))$ for all $\tilde{t}>\hat{t}$ such that $\tilde{t} \ngtr t_{0}>t$ where $\left(x\left(t_{0}\right), y\left(t_{0}\right)\right)$ is any point of the trajectory following $\hat{t}$ that is situated in the monad of the critical point of the Lienard curve $\Gamma$.

Moreover, on any such interval, essentially the trajectory satisfies the relation $\dot{y}(t)=f(x(t)) \cdot \dot{x}(t)$.

Note. The word "essentially" is transplanted from measure theory in the following sense. When we say that "essentially" the property $P$ is true on some interval $\left[t_{1}, t_{2}\right]$ we mean that it could fail only on some subset of $\left[t_{1}, t_{2}\right]$ whose measure is infinitesimal, and if it fails at some point $q$, then it fails only in "a small part" of the monad of $q$. (There exists an infinitesimal $\varepsilon_{0}>0$ such that property $P$ is false only for values of $t \approx q$ such that $|t-q|<\varepsilon_{0}$.)

Explanation. Lemma 2 states that the trajectory of (10) that finds itself in the halo of $\Gamma$, on the attracting side of $\Gamma$, must follow "the highway" until it encounters a monad of a critical point of $\Gamma$. Moreover, the slope of the trajectory cannot differ essentially from the slope of the Lienard curve $\Gamma$. 
Proof. First, let us prove that the trajectory cannot cross the highway. By our hypothesis the trajectory finds itself in the monad of $\Gamma$ on its attractive side. This means that $\dot{x}$ points towards $\Gamma$. However on $\Gamma$, the velocity $\dot{x}$ must be equal to zero, since $(a \psi(t))$ is defined while $y=F(x)$ on $\Gamma$ (see $\left.\left(13^{\mathrm{b}}\right)\right)$. $\dot{x}$ is a continuous function of $t$. Therefore, in some $\varepsilon_{0}=0$ neighborhood of $\Gamma, \dot{x}$ is infinitesimal. But $\dot{y}$ is bounded away from zero by some standard number, except, in a monad of a critical point of $\Gamma$. Thus, the trajectory will point away from $\Gamma$ along an almost vertical direction before it reaches the $\varepsilon_{0}$-neighborhood of $\Gamma$. In particular the trajectory cannot cross $\Gamma$, and, moreover, it cannot exit the highway without crossing $\Gamma$ either.

It is known that an (internal) function ${ }^{*} R \rightarrow{ }^{*} R$ which is infinite outside the monad of zero must be infinite in some sufficiently large part of the monad of zero (see Robinson [5]). Hence, in a sufficiently large part of the highway $\dot{x}$ is infinite and points towards $\Gamma$ while $\dot{y}$ is near standard. Thus, there exist some infinitesimal $\varepsilon_{1}>0$ such that the trajectory points almost horizontally towards the highway if the distance from $\Gamma$ exceeds $\varepsilon_{1}$, and the trajectory cannot leave the highway on the attractive side.

The second statement of the lemma follows from an almost identical argument. A more detailed analysis of the behavior of solutions of equation (1.1) in the halo of $\Gamma$ : $y=F(x)$ is illustrated by Lemmas 3-6.

LEMMA 3. Let $t_{1}, t_{2}$ be infinite values of the time parameter $t$ such that the segment of the trajectory $\{x=x(t), y=y(t)\}, t_{1} \leqslant t \leqslant t_{2}$, lies entirely in the halo of $\Gamma$ and $t_{2}$ is in the same galaxy as $t_{1}$ (i.e. $t_{2}-t_{1}$ is a near-standard number) and $\{x, y\}$ is not almost stationary on $\left[t_{1}, t_{2}\right]$. Let $\varepsilon>0$ denote the infinitesimal value of $y(t)-F(x(t))$ for some $t \in\left[t_{1}, t_{2}\right]$. Then it is true that the trajectory follows the highway and that on the $x$-y plane $\{y(t)-F(x(t))\}$ is located in the $\varepsilon$-galaxy of $\Gamma$ in any segment of the trajectory (on $\left[t_{1}, t_{2}\right]$ ) that does not contain a critical point of $\Gamma$. (There do not exist points $\hat{t}, \tilde{t} \in\left[t_{1}, t_{2}\right]$ such that $\left.(y(\hat{t})-F(x(\hat{t}))) /(y(\tilde{t})-F(x(\tilde{t}))) \in{ }^{*} R_{\infty}\right)$

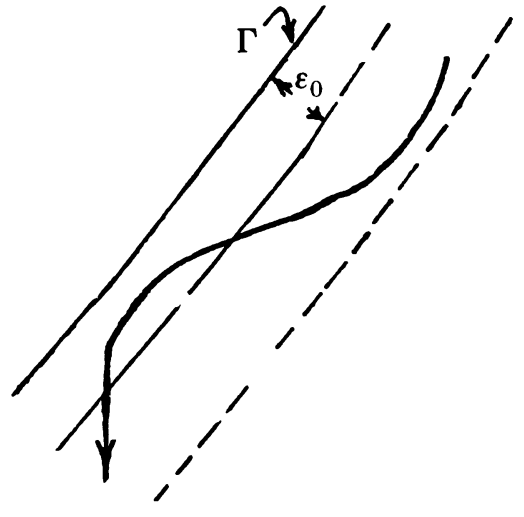

FigURE 3

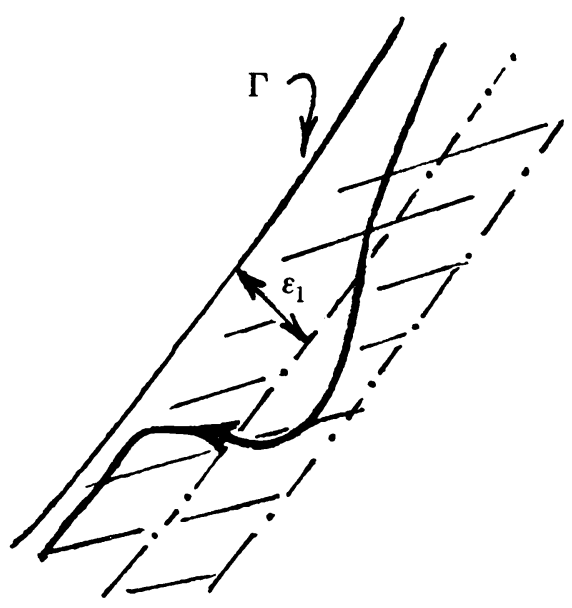

FIGURE 4 
Proof. Suppose to the contrary that for some $\hat{t}, \tilde{t} \in\left[t_{1}, t_{2}\right]$ this assertion is false. Then

$$
(y(\tilde{t})-F(x(\tilde{t}))) /(y(\hat{t})-F(x(\tilde{t}))) \in *^{*} R^{\infty} .
$$

Since the ratio $[a(\tilde{t}) \cdot F(x(\tilde{t}))] /[a(\hat{t}) \cdot F(x(\hat{t}))] \neq 0$ and is near standard, we conclude that $\dot{x}(\tilde{t}) / \dot{x}(\hat{t}) \in{ }^{*} R_{\infty}$. If $t$ does not lie in a monad of a critical point of the trajectory then $\dot{x}(t) \in{ }^{*} R_{\infty}$. Clearly, $\dot{x}(\tilde{t})-\dot{x}(\hat{t}) \in{ }^{*} R_{\infty}$. Hence, $\dot{x}(t) \in{ }^{*} R_{\infty}$ on $[\hat{t}, \tilde{t}]$. This implies that $(a(t) \cdot \psi \cdot \dot{x}) \in{ }^{*} R_{\infty}$ on $[\hat{t}, \tilde{t}]$, hence it is true that $a(t) \dot{x} \in$ ${ }^{*} R_{\infty}$ at some point $t^{1} \in[\hat{t}, \tilde{t}]$. Repeating this argument, we have $\left[a\left(\dot{x}\left(t^{1}\right)-\dot{x}(\hat{t})\right)\right] \in$ ${ }^{*} R^{\infty}$ and $\left[a \cdot\left(\dot{x}\left(t^{1}\right)-\dot{x}(\hat{t})\right)\right] \in{ }^{*} R^{\infty}$, or $a \ddot{x} \in{ }^{*} R^{\infty}$. By a similar argument we conclude that $a^{n} \ddot{x}\left(t^{n}\right) \in{ }^{*} R^{\infty}$ for any $n \in{ }^{*} N$ and $\dot{x}>1 /\left(a^{n}\right)$ for any $n \in{ }^{*} N$. To stop a "highway excursion" this must occur on an interval $\left[\hat{t}, t^{n}\right],\left|t^{n}-\hat{t}\right|<A^{n-1}$ for every infinite $n$, where $A=\max |a| \approx 0$. This is impossible, thus proving the theorem. (Observe that in a standard model $R$ no positive number $\xi$ may be found such that $\xi<A^{n}, 0<A<1$, for every $n=1,2,3, \ldots \in N$.) This statement is also true in the nonstandard model ${ }^{*} R$ for $n=1,2, \ldots \in{ }^{*} N$.

COROLlaRY. The entire part of a trajectory between an entry into the highway and the exit lies in a single $(\varepsilon)$-galaxy.

LEMMA 4. The jumps occur almost instantly (i.e. on an infinitesimal time interval). This is easy to show, since on a jump discontinuity $x$ is a fast variable, while y is slow. The jump occurs on a time interval of size $\Delta t=\left(t_{1}-t_{0}\right)$. The variation of $y$ is infinitesimal while $\Delta x / \Delta t \in{ }^{*} R_{\infty}$. Hence, $\Delta t$ must be infinitesimal if $\Delta x$ is nearstandard.

LEMma 5. After some jumps the trajectory emerges in different galaxies of $\Gamma$.

Proof. Since $\lim a(t)=0$, given any $t_{1} \in R_{\infty}$, it is true that after some time $\hat{t}>t_{1}, a(t) / a\left(t_{1}\right) \approx 0$, for all $t>\hat{t}$. Suppose that at times $t_{1}$ and $t_{2}>t_{1}$ we have $x\left(t_{1}\right) \approx x\left(t_{2}\right)$ and $y\left(t_{2}\right) \approx y\left(t_{1}\right)$, i.e. the trajectory is close to the same point of the Lienard curve at different times $t_{1}, t_{2} \in{ }^{*} R_{\infty}$, where we select $t_{2}$, so that $a\left(t_{2}\right) / a\left(t_{1}\right)=0$. Then

$$
\frac{y\left(t_{2}\right)-F\left(x\left(t_{2}\right)\right)}{y\left(t_{1}\right)-F\left(x\left(t_{1}\right)\right)} \frac{a\left(t_{1}\right)}{a\left(t_{2}\right)}=1, \quad \text { with } \frac{a\left(t_{1}\right)}{a\left(t_{2}\right)} \in * R_{\infty} .
$$

Therefore

$$
\frac{y\left(t_{2}\right)-F\left(x\left(t_{2}\right)\right)}{y\left(t_{1}\right)-F\left(x\left(t_{1}\right)\right)}=0,
$$

showing that $y\left(t_{2}\right)$ is "much closer" to $F\left(x\left(t_{2}\right)\right)$ than $y\left(t_{1}\right)$ is to $F\left(x\left(t_{1}\right)\right)$, i.e. $\left\{x\left(t_{2}\right), y\left(t_{2}\right)\right\}$ and $\left\{x\left(t_{2}\right), y\left(t_{1}\right)\right\}$ belong to different galaxies inside the monad of $\Gamma$.

Before we prove our main theorems, we need the following lemma concerning the zeros of derivatives for trajectories that follow "the highway." 
LEMMA 6. Let us denote by $\tilde{x}\left(t ; t_{0}, x_{0}\right)$ a trajectory of $(10),\left(10^{\mathrm{a}}\right)$, satisfying some initial conditions $x\left(t_{0}\right)=x_{0}$. Then for any $\hat{t} \in R_{\infty}$ we cannot have $\dot{\tilde{x}}\left(\hat{t} ; t_{0}, x_{0}\right)=0$ if the trajectory follows the highway.

Proof. Suppose that $\dot{\tilde{x}}=0$ at some value $\hat{t} \in R_{\infty}$. Then $\tilde{y}(\hat{t})=F(\tilde{x}(\hat{t}))$ and the point $\{\tilde{x}(\hat{t}), \tilde{y}(\hat{t})\}$ lies on the Lienard curve. There are two possibilities. Either (by a strange coincidence) $c(\hat{t}) \tilde{x}(\hat{t})=g(\hat{t})$, or else $\dot{y}(\hat{t})$ is a nonzero, near-standard number. In the first case

$$
\left.\frac{d y}{d x}\right|_{\Gamma}=\left.\frac{\lim \dot{y}}{\lim \dot{x}}\right|_{t \rightarrow \hat{t}}=\frac{\ddot{y}(\hat{t})}{\ddot{x}(\hat{t})}=\frac{-C(\hat{t}) \hat{x}+\dot{g}(\hat{t})}{(a \psi)^{-1} \dot{y}(\hat{t})}, \quad \text { where } \frac{d C}{d t}=c(t),
$$

but $\dot{y}(\hat{t})=0$, and so on .... After applying l'Hospital's rule a sufficient number of times we see that $d y /\left.d x\right|_{\Gamma}$ does not exists at $\hat{t}$. Therefore the trajectory does not "follow the highway" at the point $(x(\hat{t}), y(\hat{t}))$. In the second case, that is when $\dot{y}(\hat{t}) \neq 0, d y /\left.d x\right|_{\Gamma}$ does not exist because $\dot{x}(\hat{t})=0$, and the same conclusion is true.

Corollary 1. At any point of the trajectory $\tilde{x}\left(t ; t_{0}, x_{0}\right)$ at which $\dot{\tilde{x}}=0$, the trajectory "crosses the highway."

COROLlARY 2. At such a cross-over point the trajectory cannot cross from attracting to repulsive part of the Lienard curve $\Gamma$, except possibly in the monad of a critical point of $\Gamma$.

Proof. See the argument in Lemma 2.

Corollary 3. At a cross-over point the acceleration $\ddot{x}(t)$ is infinite.

THEOREM 1 (EXISTENCE OF DUCK-LIKE CYCLES). We consider equation (10) with the assumptions (10a) and $\lim _{t \rightarrow \infty} g(t)=g_{\infty}$. There exist duck-type solutions for some initial values of (10).

OUTLINE OF THE PROOF. If the Lienard curve is unbounded while there exist bounded solutions to equation (10), then the conclusion that a trajectory cannot always follow the highway is obvious. Specifically, if it did then $\dot{x}$ must vanish at some point of the trajectory, and the corollary to Lemma 6 states that the trajectory must cross "the highway", and it is easy to show that it must leave the highway altogether. Thus a "jump" must occur.

REMARK. In switching from increasing to decreasing values of $x(t)$ or vice-versa, the trajectory emerges from the attracting side of the Lienard curve to the repulsing side just before the jump occurs.

Let us demonstrate these phenomena and the existence of ducks on a specific example. We consider the standard equation

$$
\frac{d}{d t}\left[\frac{1}{t}\left(1+\cos ^{2} x\right) \dot{x}\right]+k(\cos x) \dot{x}+(A+B \sin t) x=g(t)
$$

where $A, B, k$ are some (standard) constants, $k>0$, and $g(t)$ is a continuous function on $[0, \infty]$ with $\lim _{t \rightarrow \infty} g(t)=g_{\infty}$. All conditions (10a) are fulfilled. The Lienard curve is

$$
y=k \sin x .
$$


We check the identity

$$
\dot{x}=\left[t \cdot \frac{1}{t}\left(1+\cos ^{2} x\right) /\left(1+\cos ^{2} x\right)\right] \dot{x} \equiv \dot{x}
$$

and the relation

$$
\dot{y}=g(t)-(A+B \sin t) x,
$$

that must be satisfied along a trajectory of (16).

Let us denote $c(t)=-(A+B \sin t)$. Suppose that the trajectory always follows the highway. Along the highway we have

$$
\dot{y} \simeq(\cos x) \dot{x} .
$$

This relation makes sense because $\dot{y}$ is near-standard. Obviously,

$$
\left.\frac{\dot{y}(t)}{\dot{x}(t)} \simeq \frac{d y}{d x}\right|_{\Gamma}=\cos x .
$$

There are two alternatives. Either on some points of the trajectory $\dot{x}$ is equal to zero, or else $\dot{x}$ is of constant sign, and therefore $x(t)$ is a monotone function of $t$.

We shall prove that either one of these alternatives is impossible. If for some value of $t=\hat{t}, \dot{x}(\hat{t})=0$, the relation (18) cannot be satisfied unless $\dot{y}(\hat{t})=0$. Therefore

$$
x(\hat{t})=g(\hat{t}) /(A+B \sin \hat{t})
$$

(see equation $\left.\left(13^{\mathrm{a}}\right)\right)$, and

$$
(1 / t)\left(1+\cos ^{2} x\right) \ddot{x}=0 \quad \text { at } t=\hat{t},
$$

which is possible only if $\ddot{x}(\hat{t})=0$. L'Hospital's rule implies that $\ddot{y}=0$, and

$$
\lim _{t+\hat{t}} \frac{\dot{y}}{\dot{x}}=\lim _{t \rightarrow \hat{t}} \frac{\ddot{y}}{\ddot{x}}=\lim _{t \rightarrow \hat{t}} \frac{\ddot{y}}{\ddot{x}} \text {. }
$$

Differentiating all terms of (16) once again, we obtain $\ddot{x}=0$ at $t=\hat{t}$, and so on, which leads to an easy contradiction.

Therefore at any point where $\dot{x}=0$, the relation (18) cannot be satisfied, implying that in the neighborhood of such point the trajectory of the system is not following the highway rules.

The other alternative for a trajectory obeying the highway directions is $\dot{x} \neq 0$ at any point of the trajectory. Thus $x(t)$ is a monotone function of $t$.

If $x(t)$ is bounded then there exists a number $x_{\infty}$ such that

$$
\lim _{t \rightarrow \infty} x\left(t ; t_{0}, x_{0}\right)=x_{\infty} \text {. }
$$

In nonstandard terms, this implies that for any $\tilde{t} \in R_{\infty}$ we have

$$
\hat{x}\left(\tilde{t} ; t_{0}, x_{0}\right) \approx x_{\infty} .
$$

This implies that

$$
\dot{y} \approx-c(t) x_{\infty}+g_{\infty}
$$

and

$$
y \approx C(t) x_{\infty}+g_{\infty} t+y_{0} \approx \sin x_{\infty}
$$


where $(d / d t) C(t)=c(t)$. This is impossible to satisfy unless $g_{\infty}=0, \lim _{t \rightarrow \infty} C(t)$ exists, $\lim _{t \rightarrow \infty} C(t)=C_{\infty}$, any $y_{0}$ is so chosen that

$$
y_{0}=\sin x_{\infty}-C_{\infty} x_{\infty} .
$$

This shows that the existence of a limit $\lim _{t \rightarrow \infty} x\left(t ; t_{0}, x_{0}\right)$ implies the existence of a $\operatorname{limit}_{t \rightarrow \infty} y\left(t ; t_{0}, y_{0}\right)$. This condition is impossible with our choice of $C(t)=A$ $+B \sin t$, unless $B=0$, and with any choice of $g(t)$ unless $\lim _{t \rightarrow \infty} g(t)=0$, and with any choice of $y_{0}$ that does not satisfy the relation (19).

THEOREM 2. We assume the following properties of the coefficient functions and of the inhomogeneous term in equation (10). Let

$$
\lim _{t \rightarrow \infty}[g(t)-A c(t)] \neq 0
$$

for any constant $A \neq 0$, while $\lim _{t \rightarrow \infty} a(t)=0$. Let us suppose that a trajectory $x=x\left(t ; t_{0}, x_{0}\right)$ follows the highway on a time interval $\left[t_{1}, t_{2}\right], t_{2}>t_{1} \in{ }^{*} R_{\infty}$, and the trajectory does not contain any critical points of the Lienard curve on that interval. Then $a(t)$ and $[y-F(x)]$ belong to the same $\varepsilon$-galaxy on that interval. (That is, their ratio is near-standard, while each is infinitesimal.)

Proof. It suffices if we can show that any point $\tilde{t} \in\left[t_{1}, t_{2}\right], x\left(t ; t_{0}, x_{0}\right)=\tilde{x} \neq 0$, $y\left(\tilde{t} ; t_{0}, x_{0}\right)=\tilde{y}$ on the trajectory, it is possible to find a near-standard number $\tilde{k}$ such that $a(\tilde{t})=\tilde{k}(\tilde{y}-F(\tilde{x}))$. Since the trajectory follows the highway we have along the trajectory

$$
\left.\frac{\dot{y}(\tilde{t})}{\dot{x}(\tilde{t})} \approx \frac{d y}{d x}\right|_{\{\tilde{x}, \tilde{y}\} \in \Gamma} .
$$

(Note. Some variations in the hypothesis offering a higher generality would alter that statement to $\dot{y}(\tilde{t}) / \dot{x}(\tilde{t}) \approx d y /\left.d x\right|_{\{\tilde{x}, \tilde{y}\} \in \Gamma}$ almost certainly.) That is, the velocity vector $(\dot{x}, \dot{y})$ at $(\tilde{x}, \tilde{y})$ does point in the direction almost parallel to the Lienard curve $\Gamma$. Therefore

$$
\left.\frac{d y}{d x}\right|_{\Gamma(\text { at } x=\tilde{x}, y=\tilde{y})}=\frac{(a \cdot \psi)}{(y-F)}(g-c x) .
$$

Since $[g-c(t) x]$ is near standard for all values of $t \in{ }^{*} R$ and $[g-c(t) x] \approx 0$, we conclude that there exists a near-standard number $K \approx 0$, such that in the monad of $(\tilde{x}, \tilde{y})$,

$$
\left.\frac{d y}{d x}\right|_{(\text {along } \Gamma \text { at } x=\tilde{x}, y=\tilde{y})}=K \frac{a(t) \psi(\tilde{x}(t))}{[\tilde{y}(t)-F(\tilde{x}(t))]} .
$$

Since $d y /\left.d x\right|_{(\tilde{x}, \tilde{y})} \neq 0$, because $(\tilde{x}, \tilde{y})$ is not a critical point of the Lienard curve $\Gamma$, we see that $a(t) \cdot \psi(x(t))$ and $(y(t)-F(x(t)))$ belong to the same $\varepsilon$-galaxy. This is possible only if $a(t)$ and $y(t)-F(x(t))$ belong to the same $\varepsilon$-galaxy, because $\psi(x)$ is near-standard and $\psi(x) \approx 0$.

We point out that the cycle speeds up along the jump part of the trajectory, that is the part of trajectory on which $\dot{x}^{-1} \approx 0$, because $y(t)$ is near-standard while $\lim _{t \rightarrow \infty}[a(t) \psi(x)]=0$. However, on the highway, the time interval $\Delta t$ taken 
between two points in the monad of $x_{1} \in \Gamma, x_{2} \in \Gamma$, respectively, is given by $\Delta t \approx F\left(x_{2}\right)-F\left(x_{1}\right)$. Thus, the time taken for transversing a cycle can be essentially shortened only if the length of the path is decreased in successive cycles. Consequently, if the cycles speed up, they speed up and contract towards a critical point of the Lienard curve.

\section{REFERENCES}

1. H. Poincaré, Oeuvres de Henri Poincaré, vol. VII, Gauthier-Villars, Paris, 1952.

2. V. Komkov, Continuability and estimates of solutions of $\left(a(t) \cdot \psi(x) x^{\prime}\right)+c(t) \cdot f(t)=0$, Ann. Polon. Math. 30 (1974), 125-137.

3. __ Asymptotic behaviour of non-linear differential equations via non-standard analysis. Part III, Boundedness and monotone behaviour of the equation $\left(a(t) \cdot \psi(x) x^{\prime}\right)+c(t) f(x)=q(t)$, Ann. Polon. Math. 38 (1980), 101-108.

4. A. Robinson, Introduction to model theory and to metamathematics of algebra, North-Holland, Amsterdam, 1966.

5. _ Non-standard analysis, North-Holland, Amsterdam, 1962.

6. W. A. J. Luxemberg, What is non-standard analysis, Papers in the Foundations of Mathe matics, Math. Assoc. Amer., Slaught Memorial Papers No. 13, 1966, pp. 38-67.

7. P. Cartier, Singular perturbations of ordinary differential equations and non-standard analysis, Uspehi Mat. Nauk. 39 (1984), 57-76.

8. D. P. Merkin, Gyroscopic motion, Nauka, Moscow, 1974.

9. M. Diener, Etude générique des canards, Thèse, Strasbourg, 1981.

10. Jane Cronin-Scanlon, Entrainment of frequency in singularly perturbed systems, Abstract Amer. Math. Soc. 6 (1985), Abstract 817-34-148.

11. A. L. Hodgkin and A. F. Huxley, A qualitative description of membrane current ant its application to conduction and excitation in nerves, J. Physiol. 171(1952), 500-544.

Department of Mathematics, Winthrop College, Rock Hill, South Carolina 29733 